
\title{
R Research Sourere \\ Rare Metastases in Colorectum of Infiltrating Ductal Breast Cancer: A Rare Case Report and Review
}

\section{Zhijun Zhang}

Jilin University Second Hospital

Tao Li

Jilin University Second Hospital

Yuemin Li

Jilin University Second Hospital

Dongxu Li

Jilin University Second Hospital

Kai Zhang ( $\sim$ zhangkai0628@126.com )

Jilin University https://orcid.org/0000-0002-4499-7186

\section{Case report}

Keywords: Breast ductal carcinoma, colorectum , surgery , breast cancer

Posted Date: October 20th, 2020

DOI: https://doi.org/10.21203/rs.3.rs-92091/v1

License: (c) (1) This work is licensed under a Creative Commons Attribution 4.0 International License.

Read Full License 
Rare metastases in colorectum of infiltrating ductal breast cancer: a rare case report and review

Zhijun Zhang ${ }^{1 *}$, Tao $\mathrm{Li}^{1{ }^{*}}$, Yuemin $\mathrm{Li}^{2}$, Dongxu $\mathrm{Li}^{2}$, Kai Zhang ${ }^{3}$

Zhijun Zhang1 *, Tao Li1 *, Dongxu Li ${ }^{2}$, Kai Zhang ${ }^{3}$ Department of Colorectal and Anal Surgery, the Second hospital of Jilin University, Changchun 130012, Jilin Province, China

Yuemin $\mathrm{Li}^{2}$ Department of Pathology, the Second hospital of Jilin University, Changchun 130012, Jilin Province, China

\title{
Corresponding author
}

Kai Zhang* Professor, Department of Colorectal and Anal Surgery, the Second hospital of Jilin University, No.218, Ziqiang Street, Nanguan District, Changchun City 130012, Jilin Province, China. zhangkai0628@126.com

${ }^{*}$ These authors contributed equally to this manuscript.

\begin{abstract}
Abbreviations: gastro-intestinal, Gl; Tomography-Computed Tomography, PET$\mathrm{CT}$; docetaxel + Adriamycin + cyclophosphamide, TAC regimen; Computerized Comography, CT; fluorodeoxyglucose labeled with 18F,18F-FDG; Cytokeratin 20, CK20; Special AT-rich sequence-binding protein 2, SATB2; SRY-related HMGbox, SOX; Phosphoprotein of 120 kDa, P120; Estrogen Receptor, ER; Progestogen Receptor, PR.
\end{abstract}




\section{Abstract}

Background: Breast ductal carcinoma hardly metastasizes to colorectum and the effect of surgery is controversial. We treated one case of patients with breast ductal carcinoma metastasizes to colorectum with surgery and discussed current management experience of breast cancer metastasizing to colorectum by reviewing the literature.

Case Presentation: A 37-year-old woman underwent a modified radical mastectomy three years ago for right breast cancer and developed left breast cancer with right breast cancer suspiciously metastasizing to colorectum, left ovary along with oviduct, pancreas, and left acetabulum according to positron emission tomography-computed tomography. Then she had chosen to give up further therapy but was admitted to our department complaining of shapeless feces and mucus pus and blood in stool for 2 years with the process of aggravating symptoms for recent 2 months. Colonoscopy revealed the existence of colorectal carcinoma. She received laparoscopic combined abdominal perineal resection and bilateral ovarian salpingectomy. Postoperative pathology as well as immunohistochemistry supported the origin of primary breast infiltrating ductal cancer. She remained tamoxifen therapy and was alive until she was lost to follow-up.

Conclusions: Clinicians must pay attention to any gastro-intestinal symptoms of patients with a medical history of breast cancer since the incipient symptoms of 
breast cancer metastasis to colorectum are insidious. Definite diagnosis may be difficult even by endoscopy. Surgery should be considered as a therapeutic option and definite diagnostic means combined with immunohistochemistry.

\section{Introduction}

Invasive breast carcinoma tends to disseminate even during the latency following the initial surgery. Breast cancer rarely involves in the gastrointestinal tract, of which the reported possibility is only $1 \%[1]$. The incipient symptoms of colorectal cancer are relatively dormant and the diagnosis of bowel cancer is mainly dependent on colonoscopy which is not easily acceptable. Hence, it is easy to ignore colorectal metastasis from primary breast cancer, which alerts us to pay more attention to patients complaining of gastrointestinal symptoms with a medical history of breast cancer.

\section{Case report}

A 34-year-old woman who was diagnosed with right breast cancer received a modified radical mastectomy followed by chemotherapy of TAC regimen for six cycles as well as endocrine therapy of tamoxifen for three years in 2015. Postoperational follow-up was performed as recommended and no obvious evidence shows any indication of metastases. The sole positive symptom of shapeless stool along with occasional mucus pus and blood in stool started to show up in 2016 but was unfortunately ignored. After three years, she noticed a painless mass in her left breast. Then 7 months later, she was admitted to the hospital for 
further examination. Ultrasonography revealed hypoechoic space-occupying lesions under the left nipple and fine-needle aspiration biopsy hinted breast invasive carcinoma. CT revealed left breast malignant space-occupying lesions accompanied by swollen left axillary lymph node. Then she was recommended to complete a PET-CT examination and results revealed multiple metastases to the pancreas, left acetabulum (figure 1). Increased level of 18F-FDG uptake into the rectum and left ovary, as well as left oviduct, suggested suspicious metastasis to these sites. The patients gave up any therapies and discharged.

However, the aggravating symptom of mucus and bloody stool had extremely tortured the patient and she had to resort to us. She was arranged to complete colonoscopy for further definite diagnosis and carcinomas were found on the anal margin and colon at $20 \mathrm{~cm}$ from the anus margin respectively (figure 2). Gynecological consultation was completed ahead of surgery and they would want to reevaluate that during intraoperative consultation if it would be necessary. And laparoscopic combined abdominal perineal resection and laparoscopic bilateral ovarian salpingectomy and partial vaginal resection were performed with the cooperation of a colorectal surgeon and gynecologist.

Post-operational pathology revealed invasive carcinoma in the left ovary and right oviduct as well as rectum along with its mesentery, which conformed to the morphological features of infiltrating carcinoma of the breast (figure 3). Perimesenteric lymph nodes (2/8) were also involved. The incisional margin of the 
anal canal was negative and the distance between that and carcinoma was less than $1 \mathrm{~mm}$.

Post-operational immunohistochemical staining supported that colorectal and gynecological carcinomas were both originated from breast infiltrating ductal carcinoma. Generally, the immunochemical profile for primary colorectal cancer is CK20(+), CDX2(+), SATB2(+), which was inconsistent with our results, ruling out the digestion origin. In addition, the results including GATA3(+), SOX10(-), Ecadherin(+), P120(+), CK7(+), ER(90\% positive rate, strong positive), PR $(90 \%$ positive rate, strong positive) supported infiltrating breast ductal carcinoma(luminal B1 type) (figure4).

After the surgery, the patients resumed palliative hormone therapy for the propagation of breast carcinoma. She was alive untill she was lost contact after one year of follow-up.

\section{Discussion}

Breast cancer accounts for $\sim 15 \%$ of all cancer cases in women, and ranks fifth in all causes of cancer death. Simultaneously, the overall lifetime risk for a woman is $12 \%$ [2]. Though survival has improved due to treatment advancements, incipient screening, and intensified healthy awareness, there are still $30 \%$ of breast cancers developing metastatic diseases after treatment and $5-10 \%$ are metastatic, stage IV, at diagnosis[3]. And in general, patients with metastatic breast cancer have a poor prognosis, for most can only survive 1 to 2 years[4-7]. 
Breast cancer frequently metastasizes to the liver, lungs, brain, adrenals, and, very rarely, the bones, but metastases to the $\mathrm{Gl}$ tract is quite atypical[8]. The reported rates of $\mathrm{Gl}$ metastases from breast cancer were rather low as approximately $4-18 \%$ of metastatic breast cases; furthermore, rates of colonic metastases were extremely low as 3\%[9-13]. And $5 \%-15 \%$ of breast cancer is accompanied by distant metastasis at diagnosis[10]. The reported interval between the diagnosis and the development of gastrointestinal metastasis is varying from 6 years to 30 years[4, 14, 15].

Infiltrating ductal cancer ranks the most common type of breast carcinomas while invasive lobular cancer ranks the second most common type[7]. Breast cancer rarely metastasizes to the GI tract and invasive ductal carcinoma presents only $0.2 \%$ of all cases in contrast to $4.5 \%$ of invasive lobular carcinoma[16, 17$]$. Infiltrating ductal carcinomas is far less likely to metastasize to GI[18-20], which is as reported by us. And GI tract involvement occurs more often in the stomach and small bowel when compared to colon and rectum[8].

The symptoms of metastatic tumors from primary breast cancer to the GI tract are aspecific, such as abdominal pain, diarrhea, dyschezia, bloody stools and anemia, weight loss. So it is challenging to differentiate breast cancer metastasis to the GI tract from primary digestive diseases[21]. Montagna[16] reported a case series of 40 patients with breast cancer metastasis to $\mathrm{GI}$, of which up to $20 \%$ were asymptomatic. Moreover, according to a case series of 12001 patients, $11 \%$ 
of breast cancer metastasis to GI was not diagnosed until an exploratory laparotomy was performed [15]. Besides, Ashley[22]thought surgical resection and histopathology are mandatory for diagnosis considering that colonoscopy biopsies may only capture normal mucosa. Some authors recommended more biopsies if the initial endoscopic result was negative and that it would yield a better diagnosis if assisted by endoscopic ultrasound-assisted fine needle aspiration[23].

Immunohistochemistry plays a tremendously important role in diagnosis according to the existing status of some crucial molecules. (1) GATA3: GATA3 is a transcription factor in the differentiation of breast epithelia, urothelia, and Tlymphocytes, which is expressed in over $90 \%$ of primary and metastatic ductal and lobular carcinomas of the breast while in less than $10 \%$ of primary colonic carcinoma. To be further, it is reported that strong expression of GATA3 (in $>50 \%$ tumor cells) exists in mammary (94\%), urothelial $(86 \%)$, and endometrial $(2 \%)$ adenocarcinomas instead of pulmonary, pancreatic, colonic, prostatic, and ovarian carcinomas[24]. (2) E-cadherin and P120: Breast cancer patients with a cytoplasmic expression of P120 was found to have a poor prognosis[25]. It was reported that E-cadherin loss was presented in $90 \%$ of lobular breast cancer, and some authors even regarded expression loss of E-cadherin as a unique histochemical feature of lobular breast cancer[26, 27]. Loss of E-cadherin is found in $87 \%$ of the invasive lobular carcinoma in contrast to $7 \%$ of non-lobular breast 
cancer[28]. (3) CK7 and CK20: Positive expression of CK7 and loss of CK20 generally occur in breast ductal cancer[29]. (4) CDX-2: homeobox protein CDX2 is essential to differentiation and proliferation of intestinal epithelial cells, which is specifically expressed in $96 \%$ of colorectal cancer; negative expression of CDX-2 can help to exclude colorectal origin[30]. (5) SATB2: It was reported that SATB2 was highly sensitive and specific markers for primary colorectal carcinomas[31].

The first-line treatment of primary GI malignancies is surgery while systematic therapy is preferred in breast metastasis to GI. Systematic therapy generally includes chemotherapy, radiotherapy, hormonal therapy as well as surgery according to the different status of molecules or conditions of the patient. Surgery is controversial in breast cancer metastasis to GI. And according to observatory research, the operation group did not significantly impact the median length of survival of $\mathrm{GI}$ metastasis from breast cancer compared to the non-operation group (28 vs 26 months), however, patients with only GI metastasis from breast cancer did benefit from surgery intervention [15]. Simultaneously, we cannot neglect the irreplaceable value of surgical resection plus pathology in definite diagnosis, as is mentioned before. Also, when some general indications of $\mathrm{Gl}$ operation such as obstruction, hemorrhage(anemia), and perforation occur, surgery is indispensable. So, the authors of this article believe that surgery 
options should be preserved as either substitute, adjuvant, or essential treatment means.

In conclusion, we reported an extremely rare case of infiltrating ductal breast cancer metastasis to colon and rectum with GI symptoms, which alerted us to pay enough attention to any GI symptoms of patients with a medical history of breast cancer to reach incipient diagnosis so that intervention would be given in time to prolong survival. Moreover, though the effect of surgery is limited according to observatory research, more statistical clinical trials should be included to arrive at a more reliable conclusion.

\section{Acknowledgements}

Not applicable

\section{Funding}

Funding information is not applicable

Authors' contributions Zhijun Zhang designed initiated this research. Zhijun Zhang and Tao $\mathrm{Li}$ were responsible for drafting the manuscript and sorting relevant materials and they contributed to equally to this manuscript. Yuemin $\mathrm{Li}$ and Dongxu Li made critical revisions. Yuemin Li was also helpful in drafting this manuscript. All authors read and approved the final manuscript. And all the authors approved the ranking of author contribution. 
Availability of data and materials The datasets used and/or analyzed during the current study are available from the corresponding author on reasonable request

Ethics approval and consent to participate This is a case report and doesn't contain any identifying personal information.

Patient consent for publication Patient consent has been obtained.

Competing interests The authors declare no competing interests.

1. Mistrangelo, M., et al., Obstructive colon metastases from lobular breast cancer: report of a case and review of the literature. Tumori, 2011. 97(6): p. 8004.

2. Jacobson, E.M., et al., Unexploited therapies in breast and prostate cancer: blockade of the prolactin receptor. Trends in endocrinology and metabolism: TEM, 2010. 21(11): p. 691-698.

3. Vande Berg, P., et al., A rectal metastasis of an unknown lobular breast carcinoma and its management. Acta Gastroenterol Belg, 2020. 83(2): p. 327330.

4. Benfiguig, A., et al., [Gastric metastasis of breast cancer occurring after a cancer-free interval of 30 years]. Ann Gastroenterol Hepatol (Paris), 1992. 28(4):

p. $175-7$. 
5. Schwarz, R.E., D.S. Klimstra, and A.D. Turnbull, Metastatic breast cancer masquerading as gastrointestinal primary. Am J Gastroenterol, 1998. 93(1): p. $111-4$

6. Law, W.L. and K.W. Chu, Scirrhous colonic metastasis from ductal carcinoma of the breast: report of a case. Dis Colon Rectum, 2003. 46(10): p. 1424-7.

7. Ruymbeke, H., et al., Anorectal metastasis from breast carcinoma: a case report and review of the literature. BMC Res Notes, 2018. 11(1): p. 268.

8. Gangireddy, M., et al., From the Breast to the Bowel: An Unconventional Metastatic Presentation. Cureus, 2019. 11(11): p. e6199.

9. Tsujimura, K., et al., Colonic metastasis from breast carcinoma: a case report. World J Surg Oncol, 2017. 15(1): p. 124.

10. Uygun, K., et al., Colonic metastasis from carcinoma of the breast that mimics a primary intestinal cancer. Yonsei Med J, 2006. 47(4): p. 578-82.

11. Taal, B.G., et al., The spectrum of gastrointestinal metastases of breast carcinoma: II. The colon and rectum. Gastrointestinal Endoscopy, 1992. 38(2): p. $136-141$.

12. Cormier, W.J., et al., Linitis plastica caused by metastatic lobular carcinoma of the breast. Mayo Clin Proc, 1980. 55(12): p. 747-53.

13. Klein, M.S. and P. Sherlock, Gastric and colonic metastases from breast cancer. Am J Dig Dis, 1972. 17(10): p. 881-6. 
14. Ali, W., Z.K. Mohamed, and D.J.B.J.o.M.P. Thekkinkattil, Colonic Metastasis from a Breast Carcinoma, an Unusual Colonoscopic Finding. 2016.

15. McLemore, E.C., et al., Breast cancer: presentation and intervention in women with gastrointestinal metastasis and carcinomatosis. Ann Surg Oncol, 2005. 12(11): p. 886-94.

16. Montagna, E., et al., Lobular Metastatic Breast Cancer Patients With Gastrointestinal Involvement: Features and Outcomes. Clin Breast Cancer, 2018. 18(3): p. e401-e405.

17. Haberstich, R., et al., Anal localization as first manifestation of metastatic ductal breast carcinoma. Tech Coloproctol, 2005. 9(3): p. 237-8.

18. Molina-Barea, R., et al., Lobular breast cancer metastasis to the colon, the appendix and the gallbladder. Breast Care (Basel), 2014. 9(6): p. 428-30.

19. Ambroggi, M., et al., Metastatic breast cancer to the gastrointestinal tract: report of five cases and review of the literature. Int J Breast Cancer, 2012. 2012: p. 439023.

20. Arpino, G., et al., Arpino G, Bardou VJ, Clark GM, Elledge RMInfiltrating lobular carcinoma of the breast: tumor characteristics and clinical outcome. Breast Cancer Res 6: R149-R156. Breast cancer research : BCR, 2004. 6: p. R149-56.

21. Koos, L. and R.E. Field, Metastatic carcinoma of breast simulating Crohn's disease. Int Surg, 1980. 65(4): p. 359-62. 
22. Jones, A., et al., Colonic metastasis from infiltrating ductal breast carcinoma in a male patient: A case report. Int J Surg Case Rep, 2019. 54: p. 34-38.

23. Asmar, N., et al., Gastric Metastasis Mimicking Linitis Plastica 20 Years after Primary Breast Cancer. A Case Report. J Gastrointestin Liver Dis, 2018. 27(4): p. 469-471.

24. Liu, H., et al., Immunohistochemical Evaluation of GATA3 Expression in Tumors and Normal Tissues: A Useful Immunomarker for Breast and Urothelial Carcinomas. American Journal of Clinical Pathology, 2012. 138(1): p. 57-64.

25. Venhuizen, J.H., et al., P120 and E-cadherin: Double-edged swords in tumor metastasis. Semin Cancer Biol, 2020. 60: p. 107-120.

26. Rakha, E.A., et al., Further evidence that E - cadherin is not a tumour suppressor gene in invasive ductal carcinoma of the breast: an immunohistochemical study. 2013. 62(5).

27. Walker, R.A., World Health Organization Classification of Tumours. Pathology and Genetics of Tumours of the Breast and Female Genital Organs. 2005. 46(2): p. 229-229.

28. Guiu, S., et al., Invasive lobular breast cancer and its variants: How special are they for systemic therapy decisions? Critical Reviews in Oncology/Hematology, 2014. 92(3): p. 235-257. 
29. Théraux, J., et al., Colorectal breast carcinoma metastasis diagnosed as an obstructive colonic primary tumor. A case report and review of the literature. Gastroenterol Clin Biol, 2009. 33(12): p. 1114-7.

30. Bayrak, R., H. Haltas, and S. Yenidunya, The value of CDX2 and cytokeratins 7 and 20 expression in differentiating colorectal adenocarcinomas from extraintestinal gastrointestinal adenocarcinomas: cytokeratin 7-/20+ phenotype is more specific than CDX2 antibody. Diagn Pathol, 2012. 7: p. 9.

31. Lin, F., et al., Cadherin-17 and SATB2 are sensitive and specific immunomarkers for medullary carcinoma of the large intestine. Arch Pathol Lab Med, 2014. 138(8): p. 1015-26.

Fig.1. PET-CT. A. 18F-FDG uptake increased in colon. B. 18F-FDG uptake increased in left acetabulum. C. 18F-FDG uptake increased in pancreas.

Fig.2. Colonoscopy. A. Hyperplasia of the anal margin. B. An ulcerated swelling with a circumferential elevation of the mucosa at $20 \mathrm{~cm}$ from the anus.

Fig.3. HE staining ( $\times 100)$. A. Breast cancer metastases to ovary (black arrow) and normal ovarian stroma (red arrow). B. Breast cancer metastases to oviduct (black arrow) and normal oviduct. C. Breast cancer metastases to the bowel (black arrow) and normal intestinal gland (red arrow).

Fig.4. Immunohistochemical staining. A. GATA3(+), B. CK7(+): supporting breast cancer origin $(\times 100)$. C. E-Cadherin $(+)$, D.P120(+): supporting breast ductal carcinoma $(\times 200)$. E. SATB2(-), F. CDX2(-), G.CK20(-): excluding primary colorectal cancer. 



\section{Figures}
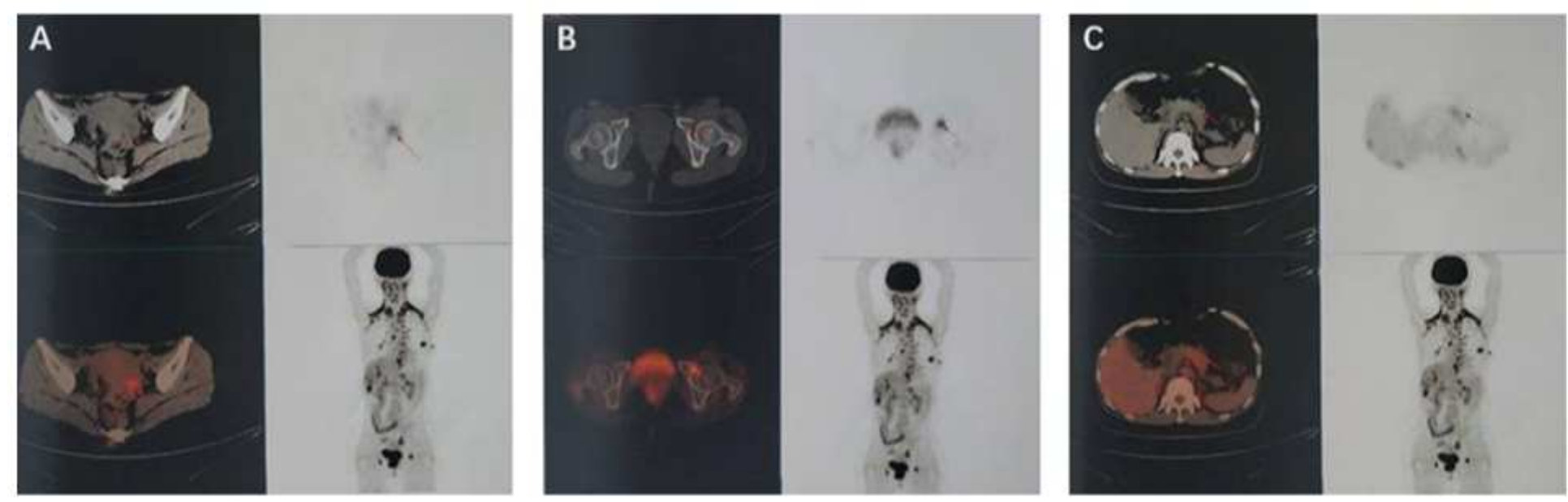

\section{Figure 1}

PET-CT. A. 18F-FDG uptake increased in colon. B. 18F-FDG uptake increased in left acetabulum. C. 18FFDG uptake increased in pancreas.
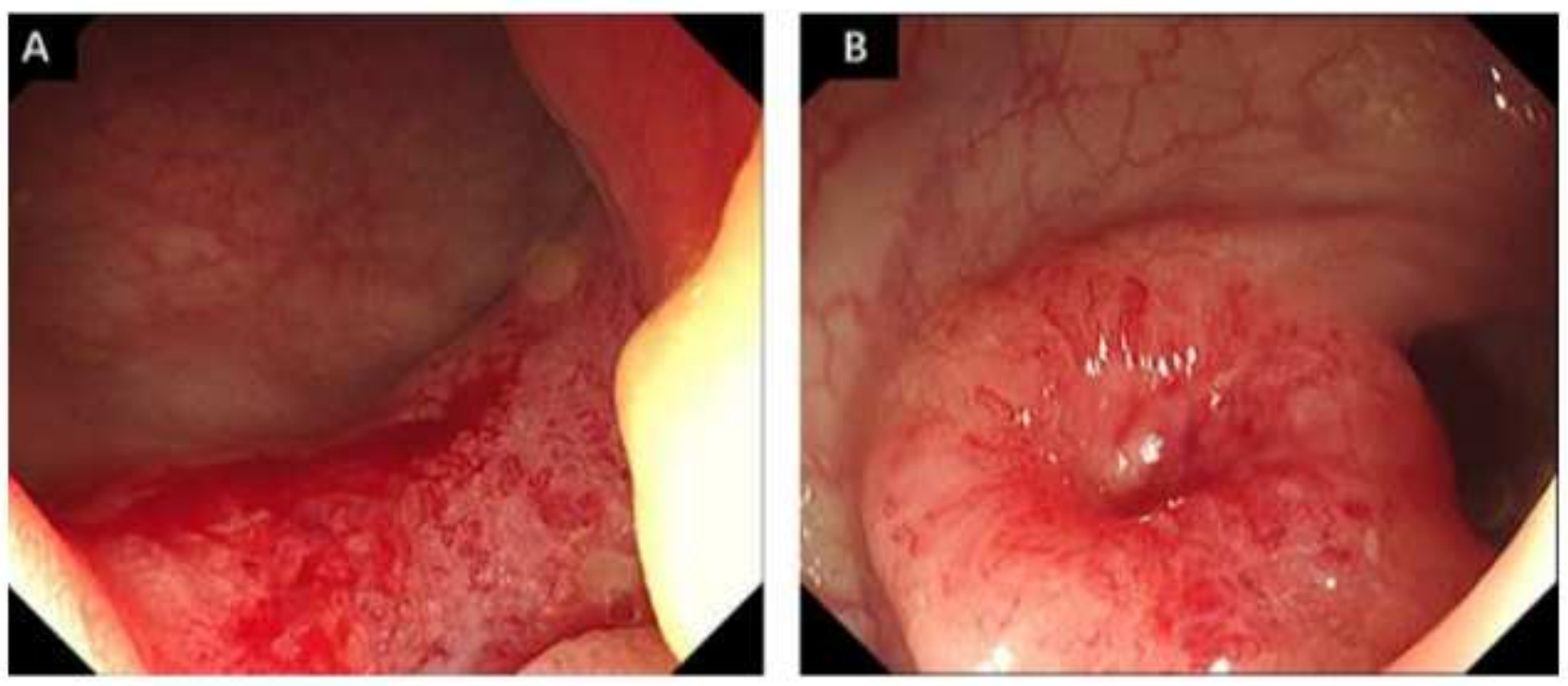

Figure 2

Colonoscopy. A. Hyperplasia of the anal margin. B. An ulcerated swelling with a circumferential elevation of the mucosa at $20 \mathrm{~cm}$ from the anus. 

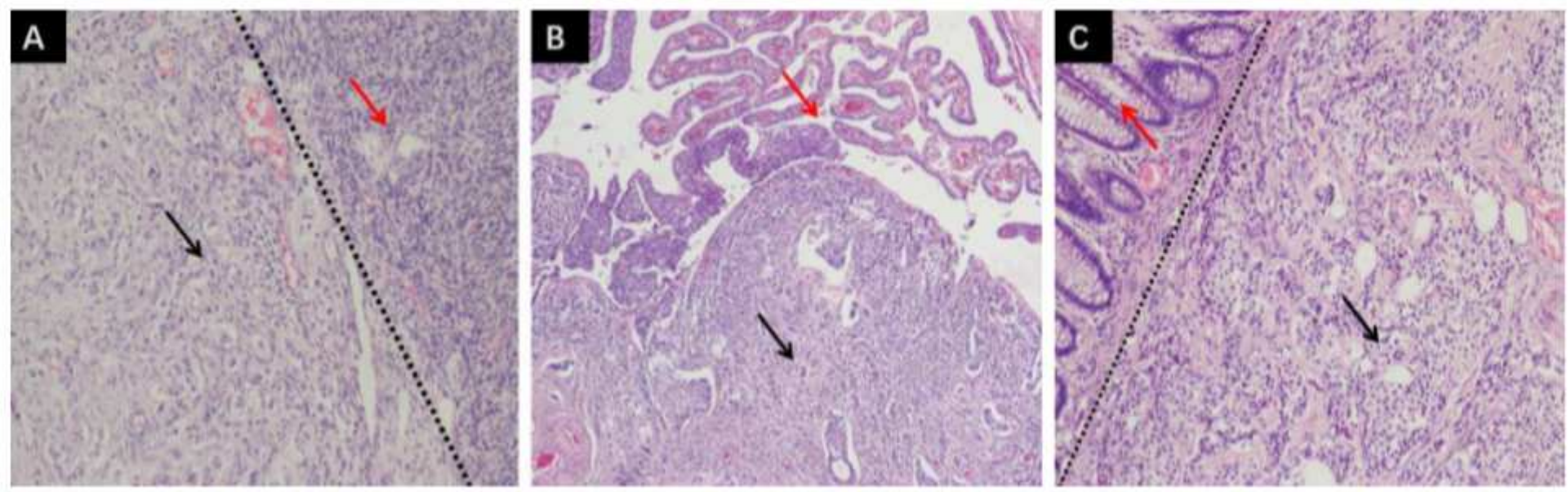

\section{Figure 3}

HE staining $(\times 100)$. A. Breast cancer metastases to ovary (black arrow) and normal ovarian stroma (red arrow). B. Breast cancer metastases to oviduct (black arrow) and normal oviduct. C. Breast cancer metastases to the bowel (black arrow) and normal intestinal gland (red arrow).
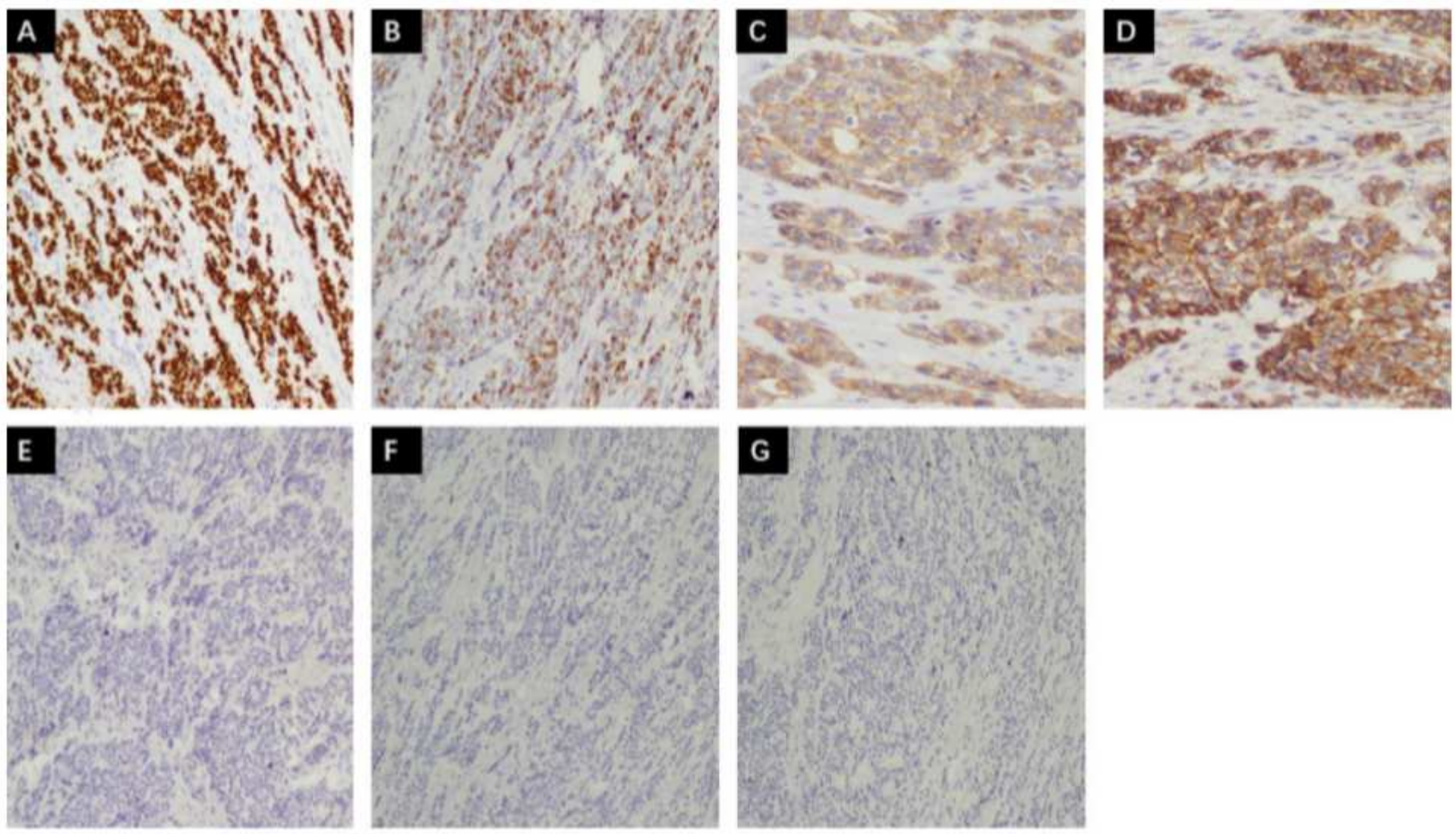

\section{Figure 4}

Immunohistochemical staining. A. GATA3(+), B. CK7(+): supporting breast cancer origin (x100). C. ECadherin(+), D.P120(+): supporting breast ductal carcinoma(×200). E. SATB2(-), F. CDX2(-), G.CK20(-): excluding primary colorectal cancer. 